\title{
Characterization of the Anopheles gambiae octopamine receptor and discovery of potential agonists and antagonists using a combined computational-experimental approach
}

Kevin W Kastner ${ }^{1 \dagger}$, Douglas A Shoue ${ }^{3+}$, Guillermina L Estiu ${ }^{2}$, Julia Wolford ${ }^{3}$, Megan F Fuerst ${ }^{3}$, Lowell D Markley ${ }^{2}$, Jesús A Izaguirre ${ }^{1 \dagger}$ and Mary Ann McDowell ${ }^{3^{*}+}$

\begin{abstract}
Background: Octopamine receptors (OARs) perform key functions in the biological pathways of primarily invertebrates, making this class of G-protein coupled receptors (GPCRs) a potentially good target for insecticides. However, the lack of structural and experimental data for this insect-essential GPCR family has promoted the development of homology models that are good representations of their biological equivalents for in silico screening of small molecules.
\end{abstract}

Methods: Two Anopheles gambiae OARs were cloned, analysed and functionally characterized using a heterologous cell reporter system. Four antagonist- and four agonist-binding homology models were generated and virtually screened by docking against compounds obtained from the ZINC database. Resulting compounds from the virtual screen were tested experimentally using an in vitro reporter assay and in a mosquito larvicide bioassay.

Results: Six An. gambiae OAR/tyramine receptor genes were identified. Phylogenetic analysis revealed that the OAR (AGAP000045) that encodes two open reading frames is an a-adrenergic-like receptor. Both splice variants signal through CAMP and calcium. Mutagenesis analysis revealed that D100 in the TM3 region and S206 and S210 in the TM5 region are important to the activation of the GPCR. Some 2,150 compounds from the virtual screen were structurally analysed and 70 compounds were experimentally tested against AgOAR45B expressed in the GloResponse ${ }^{\text {TM } C R E-l u c 2 P ~ H E K 293 ~ r e p o r t e r ~ c e l l ~ l i n e, ~ r e v e a l i n g ~} 21$ antagonists, 17 weak antagonists, 2 agonists, and 5 weak agonists.

Conclusion: Reported here is the functional characterization of two An. gambiae OARs and the discovery of new OAR agonists and antagonists based on virtual screening and molecular dynamics simulations. Four compounds were identified that had activity in a mosquito larva bioassay, three of which are imidazole derivatives. This combined computational and experimental approach is appropriate for the discovery of new and effective insecticides.

Keywords: Anopheles gambiae, Octopamine receptor, Homology modelling, Virtual screening, Molecular dynamics, G-protein coupled receptors

\footnotetext{
* Correspondence: mcdowell.11@nd.edu

${ }^{\dagger}$ Equal contributors

${ }^{3}$ Department of Biological Sciences, Eck Institute for Global Health, University

of Notre Dame, Notre Dame, IN, USA

Full list of author information is available at the end of the article
} 


\section{Background}

Despite decades of research and multiple initiatives, vectortransmitted diseases remain a major public health threat throughout the world. Blood-feeding insects transmit some of the most debilitating infections known to mankind, including malaria, lymphatic filariasis, yellow fever, and leishmaniasis. Malaria is the most deadly vector-borne disease in the world, threatening about $40 \%$ of the world's population and causing nearly one million deaths, primarily in African children [1]. Anopheline mosquitoes are the primary vectors of Plasmodium parasites, the causative agents of malarial disease to humans. Although the implementation of artemisinin-based combination therapies in the mid-1990s helped to reduce the global mortality and morbidity due to malaria, vector control has been the cornerstone of malaria control programs, primarily through the use of insecticide-treated bed nets and to a lesser extent, indoor residual spraying. The recent emergence of artemisinin resistance in Plasmodium falciparum [2] underscores the role of vector control for reducing the devastation of malaria world-wide. Unfortunately, insecticide resistance is increasing globally, requiring development of more effective insecticides [3].

Octopamine and tyramine are related biogenic monoamines that act as neurohormones, neuromodulators and neurotransmitters in invertebrates $[4,5]$, performing essential functions and modulating many crucial physiological processes. These processes include learning and memory, locomotion, feeding behaviours, the pheromone response, and cardiac function. In addition, octopamine plays this physiological role in invertebrates only $[4,5]$. These reasons make octopamine receptors (OARs) excellent targets for developing new and safer insecticides.

Formamides such as dimethylchlordimeform and amitraz mimic octopamine and provide broad-spectrum insecticidal activity [6]. These insecticides have been used in veterinary practice to control ticks for nearly 40 years, however the quest for novel control compounds that target OARs has been relatively ineffective. However, recent technological developments in screening protocols, however, may reverse this trend [4].

OARs are G-protein coupled receptors (GPCRs), a family of seven transmembrane (TM) receptors that are involved in many diseases and are the target of approximately 30\% of all modern medicinal drugs $[7,8]$. GPCRs are called such because they interact with G-protein trimeric complexes, the three main types being $G_{\alpha s}, G_{\alpha i}$, and $G_{\alpha q}$.

Few GPCR crystal structures have been elucidated due to the complexity of these proteins as well as their location in lipid membranes. While the limited crystal structures that have been solved generally have low sequence identity (for example, the sequence identity of the $\beta_{2}$-adrenergic receptor and rhodopsin is below 20\%), the 3D structures of their TM regions are found to be very similar. Large structural differences are generally found in the loop regions, where location and secondary structure between the receptors can deviate [9]. Realizing this characteristic, it is hypothesized that $3 \mathrm{D}$ molecular modelling using an existing GPCR model as a template, and performing a simulation on the resulting model, will fix major variations from the existing model and prepare a novel GPCR for virtual screening.

GPCRs are activated via agonists docking to the interior of the receptor near the extracellular side. To date, three residues have been found to be important for the activation of most GPCRs. These are an aspartic acid in the third TM region (TM3) and two of three closely grouped serine residues found in TM5. The endogenous biogenic amine agonist appears to hydrogen bond via its amine group and its hydroxyl groups to the aspartic acid and serines of the GPCR, respectively [10-12].

Studies have been conducted studying OARs of other insects, including the honey bee [13], silkworm [14], cockroach [15], and fruit fly [16,17]. These have mostly been molecular and functional characterizations of the receptor, though many studies have yielded the effects of common GPCR agonists and antagonists on their respective OARs. Here, an OAR from Anopheles gambiae mosquitoes was characterized and novel agonists and antagonists were discovered through molecular dynamics (MD) simulations and virtual screening, followed by larval bioassays with candidate compounds.

\section{Methods}

\section{Insects and materials}

Anopheles gambiae (strain PEST) mosquitoes were raised and maintained in an environmental chamber at $26^{\circ} \mathrm{C}$, $85 \%$ relative humidity, with a 16-hour light, eight-hour dark cycle including a one-hour dusk/dawn period [18]. Larvae were fed daily a 2:1 mixture of fish pellets: brewer's yeast, that had been finely ground [19]. DLoctopamine, tyramine, dopamine, naphazoline, clonidine, serotonin, chlorpromazine, cyproheptadine, promethazine, all hydrochloride salts, and tolazoline a benzylimidazoline salt, were obtained from Sigma-Aldrich. Metoclopramide hydrochloride was obtained from MP Biomedical. Compounds identified in the virtual screen were purchased from Princeton BioMedical, ChemDiv, Chembridge and Enamine and tested in vitro against AgOAR45B expressed in the GloResponse ${ }^{\mathrm{Tm}} \mathrm{CRE}-$ luc2P HEK293 reporter cell line and in larval bioassays.

\section{Expression analysis of $A g O A R 45 A$ and $A g O A R 45 B$}

Total RNA was isolated from five different $A n$. gambiae immature stages (L1-P), adult females and males, adult female heads only, and adult female abdomen/thorax using the RNeasy Mini Kit (Qiagen). The DNase (Fermentas)treated RNA was used to generate cDNA using Superscript 
III (Invitrogen) and oligo ( $\left.\mathrm{dT}_{12-20}\right)$, according the manufacturer's recommendations. Quantitative PCR (qPCR) was performed using SYBRGreen (ABI), an ABI 7900 RT-PCR system and $200 \mathrm{ng}$ of cDNA per sample, a final concentration of $0.15 \mathrm{M}$ of each primer, and an annealing temperature of $60^{\circ} \mathrm{C}$. Primer sets used for expression analysis were: Ag10592 forward- CACCATCGAACACAAAGTTGACA CTT; Ag10592 reverse- CGAACGTAACGTCACGGCCA; Ag45A\&B forward- GGGTACGTCGTCTACTCAGCCC TC; Ag45A reverse- TGTATCCGCAGCGTTAGCCGAT TG; Ag45B reverse- CGAGATTGTTCTTGCCACCTTTG GTG. The 40S Ribosomal protein subunit 7 (AGAP01592) was used as an internal control. Reactions for each gene and for the control used were carried out in triplicate. Relative expression levels of each gene was determined by the $\Delta \Delta C_{T}$ method, where relative expression is expressed as a fold difference relative to whole females and expressed as $2^{-\Delta \Delta C T}$. The following formula was used: $\Delta \Delta \mathrm{C}_{\mathrm{T}}=\Delta \mathrm{C}_{\mathrm{T} \text { (stage or }}$ condition) $-\Delta \mathrm{C}_{\mathrm{T} \text { (Females) }}$ and $\Delta \mathrm{C}_{\mathrm{T}}=\mathrm{C}_{\mathrm{T}}$ (gene of interest) $\mathrm{C}_{\mathrm{T} \text { (40S RNA). }}$

\section{Heterologous expression of AgOAR45B octopamine receptor}

Total RNA was isolated from heads of three-day old adult females using RNeasy Mini Kit (Qiagen). cDNA was synthesized using SuperScript III (Invitrogen), and used as a template for PCR amplification of the AgOAR45A and $A g O A R 45 B$ genes. Insertion of the coding sequences into the Sgfl and PmeI sites of the pF9a CMV hRluc-neo vector (Promega) was performed by digestion of a fragment amplified by the following primers: Ag45forwardTAAAGCGATCGCCATGAACGAGTCGGAGTGTGCC; Ag45Areverse- TTGTGTTTAAACTCTCGAGTCGGACAG GTCGC; Ag45Breverse- CGCGGTTTAAACTCTGAACAC ACCACCGACGA. Primers were constructed based on the annotated sequence of the AGAP000045 gene (VectorBase, Protein ID: AGAP000045-PA and -PB [20].

GloResponse ${ }^{\mathrm{TM}} \mathrm{CRE}-$ luc2P HEK293 reporter cell line (Promega) were maintained as adherent culture at $37^{\circ} \mathrm{C}$, $5 \% \mathrm{CO}_{2}$ in DMEM (Invitrogen) supplemented with $10 \%$ fetal calf serum (Atlanta), and $50 \mathrm{mg} / \mathrm{ml}$ hygromycin B. Transfection of cells was carried out using the Amaxa Nucleofector kit per the manufacturer's instructions. Control transfections were performed using a pF9A plasmid with the barnase (Bacterial Ribonuclease) gene removed as suggested by the manufacturer (Promega). Stable lines

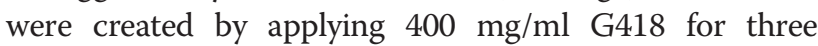
weeks. Stable clones of AgOAR45B expressing HEK293 reporter cells were created through two rounds of limiting dilution cloning.

\section{cAMP assay}

Intracellular cAMP increase was monitored through a CRE-luc reporter construct in HEK293 cells (Promega).
Stable cell lines were plated at a density of $4 \times 10^{4}$ cells per well in white 96-well assay plates (Corning, Cat. \#3917). Cells were immediately treated with the various compounds, and returned to the incubator for four hours before being assayed. cAMP was quantified through luciferase production using the Dual-Glo luciferase assay kit (Promega) following the manufacturer's instructions. Luciferase units were normalized to the cell number using the internal Rluc construct in the pF9A expression plasmid.

\section{Intracellular $\mathrm{Ca}^{++}$assay}

Stable cell lines expressing AgOAR45A or AgOAR45B were plated in black well, clear bottom, 96-well assay plates (Greiner Bio-One, Cat. \#655090) at $4 \times 10^{4}$ cells per well. Cells were allowed to grow overnight before being assayed. Cells were preloaded with Fluo-4 NW (Molecular Devices), and carried out per the manufacturer's instructions. Fluorescence was monitored before and after addition of compounds using the Flexstation3 (Molecular Devices), at two-second intervals for 120 seconds.

\section{Site-directed mutagenesis of AgOAR45B}

The $A g O A R 45 B$ gene was subcloned into a TA vector (Invitrogen), according to the manufacturer's instructions. Site-directed mutagenesis of $A g O A R 45 B$ was carried out using Quick Change Lightning Kit (Agilent). Primers (IDT) were designed to introduce single amino acid changes (see Additional file 1). Double mutants were created through two rounds of mutagenesis. Mutations were confirmed through automated sequencing (ND Genomics Core Facility). Mutant genes were then excised and moved to the pF9A expression vector as described above.

\section{Membrane preparation and radioligand binding assay}

Cells in T75 flasks were washed with $10 \mathrm{ml}$ of PBS, removed by scraping, centrifuged at $500 \mathrm{~g}$, and then resuspended in $5 \mathrm{ml}$ of lysis buffer, $50 \mathrm{mM}$ Tris-Cl pH 7.4. Cells were incubated on ice for $10 \mathrm{~min}$ and homogenized with a Dounce homogenizer with 30 strokes. The homogenate and $5 \mathrm{ml}$ wash with lysis buffer were then centrifuged at $23,000 \mathrm{~g}$ for $30 \mathrm{~min}$ at $4^{\circ} \mathrm{C}$ to pellet crude membranes. The resulting pellet was re-suspended in 50 mM Tris-Cl pH 7.4, 5 mM MgCl2, 0.5 mM EDTA pH 7.4 and quantified by micro BCA assay (Thermo).

Radioligand binding assays were carried out using ${ }^{3} \mathrm{H}$-Yohimbine to determine binding affinity of the octopamine receptor with different compounds. Isolated membranes, $30 \mu \mathrm{g}$ per well, were incubated in the presence of $16 \mathrm{nM}{ }^{3} \mathrm{H}$-Yohimbine (American Radiolabeled Chemicals) in binding buffer, and various concentrations of compounds. The final reaction volume was $125 \mathrm{ml}$ per well in a 96-well assay plate. One-hundred $\mathrm{mM}$ clonidine was used to determine non-specific binding in each 
experiment. After a two-hour incubation, membranes were collected and washed on filter mats, pretreated with $0.3 \%$ polyethylenimine (Sigma) using a Brandel 96-well harvester and counted in a Perkin Elmer Microbeta counter.

\section{Larval bioassay}

Dose response curves were made to determine the $\mathrm{LD}_{50}$ for some compounds against three-day old Aedes aegypti larvae. Ten larvae were put in each well of a 12-well plate, with each well containing a different concentration of compound in water. Three replicate wells were made for each experiment and the curves performed three separate times. Control wells were included in each experiment, which contained only DMSO in water. Plates were incubated 24 hours in standard insectary conditions. Mortality was determined after 24 hours.

\section{Project workflow}

A computational-experimental workflow (see Additional file 2), similar to the computational approach described by Yarnitzky et al. [21] was utilized. The method was as follows: 1) homology models, both inactive (antagonistbased) and active (agonist-based) conformations, were created using the fragment-based method I-TASSER (Iterative Threading Assembly Refinement) [22-24] as well as surrounded by lipids, water, and ions using visual molecular dynamics (VMD) [25]; 2) a preliminary virtual screen with one of two compound test sets (composed of either GPCR agonists or antagonists whose activity on AgOAR45B was experimentally determined using an AgOAR45B reporter assay) was performed and a set of top-ranked positions of the most were chosen and used in MD simulations with the proper active (agonist-binding) and inactive (antagonist-binding) protein conformations; 3) MD simulations were performed until stable ligand positions were obtained, then an additional virtual screen with the test set was performed on each resulting protein conformation and the results were analysed manually. Step 3 was repeated until the ligand positions were stabilized in the protein even after ten additional nanoseconds (ns) of simulation; 4) final conformations were then used to build grids which were subjected to virtual screening using the ZINC library; and, 5) the resulting compounds were analysed and compounds with differing structural characteristics were purchased and experimentally tested in vitro.

\section{Homology modelling}

Both initial inactive (antagonist-based) and active (agonistbased) conformations of AgOAR45B were generated using the I-TASSER online server [26]. For the inactive conformation, a structure was obtained that was built based on many GPCR antagonist-bound and inverse agonistbound conformations, primarily a crystal structure of $\beta_{2-}$ adrenergic receptor with partial inverse agonist carazolol bound [PDB:2RH1]. For the active conformation, a structure was obtained that was built based on the crystal structure of the $\beta_{2}$-adrenergic receptor- $G_{\alpha s}$ protein complex with high affinity agonist BI-167107 bound [PDB:3SN6]. Both conformations were obtained using standard ITASSER settings. In the case of the active conformation of AgOAR45B, 3SN6 (chain R) was used as a template to generate the model. Root-mean-square deviations (RMSDs), as a measure for protein stability, were calculated using the VMD 1.9 RMSD Trajectory Tool [25]. AgOAR45B has a $24 \%$ sequence identity ( $35 \%$ similarity) to the $\beta_{2}$-adrenergic receptor (see Additional file 3). The active site residues were determined from analyzing the inactive and active $\beta_{2}$ adrenergic receptor's crystal structures and observing residues that interacted or had the potential to interact with the crystal structures' bound ligands $[27,28]$. The active site sequence identity is much higher at $67 \%$ (73\% similarity).

\section{Molecular dynamics}

Using VMD 1.9, each virtual representation of the protein was first embedded in a large 1-palmitoyl-2-oleoylphosphatidylcholine (POPC) lipid bilayer, removing any lipid that overlapped with the protein. The virtual proteinmembrane system was then solvated with TIP3P water molecules, and neutralized by virtually adding $\mathrm{KCl}$ up to $400 \mathrm{mM}$. Initially, CHARMM 27 parameters [29] were assigned to all molecules using VMD 1.9 to enable the addition of the lipid bilayer, water and ions. However, once each virtual system (both active and inactive conformation) was prepared and its respective ligand (octopamine for the active conformation and promethazine for the inactive conformation) was ready to be added, AMBER gaff and ff03.r1 parameters were assigned to the ligand and the rest of the molecules, respectively, using Amber 11 tleap [30-32]. The AMBER force field was chosen as it allows the generation of parameters for the ligand using the antechamber module [33]. A disulphide bridge was added between the residues of Cys93 and Cys194, as this bridge also existed in the template PDBs.

Each complete virtual system consisted of the respective conformation of AgOAR45B embedded to a large POPC bilayer with 168 lipid molecules. In each virtual system, all residues were at the normal protonation state for physiological $\mathrm{pH}$. In addition, the antagonist- bound system contained 171 potassium ions, 204 chloride ions, and 22,525 water molecules for a total of 99,706 atoms (measured $95 \times 96 \times 128 \AA$ ), while the agonist-bound system contained 172 potassium ions, 205 chloride ions, and 22,654 water molecules for a total of 100,077 atoms (measured $101 \times 92 \times 127 \AA$ ). . Before MD simulations the systems were equilibrated using $120 \mathrm{CPU}$ cores as follows: 1$) \mathrm{MD}$ of lipid tails for 500 picoseconds (ps) [time step $=2$ femtoseconds (fs)] with protein, ligand, lipid head groups, water, 
and ions kept fixed; 2) equilibration for lipids, water and ions for $500 \mathrm{ps}$ (time step $=2 \mathrm{fs}$ ) with harmonic constraints on the protein and ligand; 3) equilibration of the entire system for 500 ps (time step $=2 \mathrm{fs}$ ) with no molecular constraints. After equilibration, 20-30 ns of MD simulation were performed using $504 \mathrm{CPU}$ cores in two to three 10 ns increments, with time step $=2 \mathrm{fs}$ and trajectory data being collected every 200 ps. The equilibration and simulation steps were run using NAMD 2.8 [34] on the highperformance computing cluster Kraken [35].

\section{Virtual screen preparation}

All virtual screening jobs were run on their respective 'protein only' homology models (i.e., containing no lipids, waters or ions). The proteins were prepared by first running the Protein Preparation Wizard workflow [36,37], and then grids were generated using Glide's Receptor Grid Generation application, each found in Schrodinger Suite 2011's Maestro [38]. To obtain initial ligand poses used in grid generation, each of the conformations were first overlapped in PyMOL [39] with the top templates used by I-TASSER for their creation (2RH1 for the inactive conformation and 3SN6 for the active conformation) and the positions of the ligands found in each of the templates were first saved as a PDB file, then added using Schrodinger Suite 2011's Maestro to their respective AgOAR45B conformation (2RH1 partial inverse agonist CAU was used for the inactive conformation and 3SN6 agonist 30G was used for the active conformation) to denote the active site of the protein. The ligand added to each of the homology models was used as the centroid of the grid, determining the area in which the libraries of compounds should be docked. No constraints were used.

\section{Virtual screening with known GPCR ligands}

The inactive and active conformations of AgOAR45B with the added ligands were used to build the grids, which were then run in a virtual screen using Schrodinger's Glide software [40-43] against known GPCR antagonists and agonists, respectively. The original compound structures used in the two test sets were downloaded from the NIH's PubChem website. One of the test sets contains known GPCR agonists: synephrine, cinnamic acid, clonidine, demethylchlordimeform, dopamine, eugenol, histamine, naphazoline, norepinephrine, octopamine, phentolamine, serotonin, tolazoline, transanethole, and tyramine. The other containing known GPCR antagonists: rauwolscine, demethylchlodimeform, 8-hydroxymianserin, amitriptyline, antazoline, chlorpromazine, cyproheptadine, desipramine, desmethylmianserin, dihydroergotamine, gramine, imipramine, maroxepin, metoclopramide, mianserin, phentolamine, prazosin, promethazine, propranolol, triprolidine, and yohimbine. Each of the test sets was then prepared using Schrodinger Maestro's LigPrep [44] to generate different potential protonation states at $\mathrm{pH}$ range 7-8, tautomers and ring conformations. After each of the test sets was docked to its respective AgOAR45B conformation, top pose positions for the antagonist promethazine and the agonist octopamine were saved and later added to the inactive (antagonist-bound) and active (agonist-bound) conformations, respectively. These protein conformations with docked ligands were then used as the starting position for the MD simulations.

\section{Virtual screening with ZINC library}

The library used in the docking contained drug-like compounds from the ZINC online database [45]. Compounds were prepared using Schrodinger Maestro's LigPrep to generate different potential protonation states at $\mathrm{pH}$ range 5-9, tautomers and ring conformations. The final library contained approximately 12 million compounds. It was split into five sublibraries ( 2.4 million compounds per sublibrary) for the first run of high-throughput virtual screening.

The library was screened against five grids of AgOAR45B (two from the antagonist-bound conformations and three from the agonist-bound conformations), built from the receptor positions after $20 \mathrm{~ns}$ of MD simulation each. Virtual screening using the ZINC library was performed using Schrodinger's Glide software in three steps: 1) each of the five sublibraries was filtered using high-throughput virtual screening and the top 30,000 compounds in each sublibrary were saved and recombined making a library containing 150,000 compounds; 2) this new library was then filtered further using standard precision virtual screening and the top 15,000 compounds were saved; and, 3) this new library was then filtered one more time using extra precision virtual screening and the top 1,500 compounds were saved and analysed, considering the score, the relevant interactions in the active site pocket and diversity in the sampling for further testing.

\section{Results}

\section{Molecular characterization of Anopheles gambiae} octopamine receptors

Anopheles gambiae OAR and the closely related tyramine receptor (TyrR) genes were identified by homology searching utilizing an OAR sequence (DmOamb) from Drosophila melanogaster. Seven possible An. gambiae OAR/TyrR genes were identified in VectorBase, AGAP000045, AGAP002519, AGAP002888, AGAP002886, AGAP004034, AGAP013324, and AGAP011698. One locus, AGAP011698, contained only approximately one-half of the predicted coding sequence of DmOamb and, although likely encoding an OAR or TyrR, was eliminated from further analysis. The closest homologue to DmOamb was AGAP000045. Similar to the DmOamb locus that encodes for two alternatively spliced genes, DmOamb-D and DmOamb-B, the AGAP000045 
locus encodes two open reading frames (ORF), AGAP00 0045_OARA (AgOAR45A) of 1,779 bp and AGAP00 0045_OARB (AgOAR45B) of 1,773 bp, that encode putative proteins consisting of 592 and 590 amino acids, respectively. The splice variants have a deduced amino acid identity of $56.1 \%$, with the first three exons being identical and the fourth and largest $(1,046 \mathrm{bp}$ for $A g O A R 45 A$ and 1,040 bp for $A g O A R 45 B)$ exons being alternatively spliced (see Additional file 4A and B). The hydropathy plot of both AgOAR45A and AgOAR45B revealed seven hydrophobic domains, indicative of seven transmembrane-spanning regions typical of GPCRs (see Additional file $4 \mathrm{C}$ ). In addition, both predicted proteins contain the GPCR characteristic aspartic acid (D) in TM3 and two serine (S) residues in TM5.

\section{Phylogenetic analysis}

A phylogenetic tree was constructed using the ClustalX 2.1 and the neighbour joining method of 21 OARs and TyrRs from An. gambiae, Ae. aegypti, Bombyx mori, Culex quinquefasciatus, and D. melanogaster. The AGAP000045 predicted proteins group with the $\alpha$-adrenergic-like receptors, such as DmOamb (Figure 1), exhibiting a 34.9-37.5\% deduced amino acid identity similarity with the DmOamb splice variants, a $20.7-21 \%$ similarity to DmOct-TyrR, a $10.8-18.2 \%$ similarity with the Drosophila $\beta$-adrenergiclike receptors, and a $19.5-21.7 \%$ similarity to the Drosophila TyrR. AGAP002886 and AGAP002888 likely encode for $\beta$-adrenergic-like receptors, AGAP002519 an OAR/ TyrR and AGAP004034 and AGAO013324 each a TyrR (Figure 1).

\section{Expression of AgOAR45B}

Quantitative PCR was performed to assess the expression pattern of $A g O A R 45 A$ and $A g O A R 45 B$ at different developmental stages and tissues of An. gambiae mosquitoes. Both genes were expressed in all life stages, with the lowest expression being in the larval stages. Importantly, both $A g O A R 45 A$ and $A g O A R 45 B$ were expressed in female heads (Figure 2).

\section{Functional characterization of AgOAR45A and AgOAR45B}

To functionally characterize AgOAR45A and AgOAR45B, cDNAs of both proteins were separately cloned and stably expressed into the GloResponse ${ }^{\mathrm{mm}}$ CRE-luc2P HEK293 reporter cell line. Compared to the reported sequence in VectorBase, the cloned AgOAR45A gene contained five silent mutations and a 6-bp deletion resulting in two fewer glutamines (Q426 and Q427) in a glutamine repeat region. The cloned $A g O A R 45 B$ gene differed from the VectorBase sequence with two silent single nucleotide polymorphisms (SNPs), two SNPs resulting in a valine $(\mathrm{V})$ at position 45 instead of an alanine (A) and a switch from an arginine (R) at position 403 to a glutamine (Q), a 3-bp deletion resulting in the lack of one histidine in a histidine repeat region and an insertion of a valine $(\mathrm{V})$ at the end of the sequence, due to the cloning design.

Both genes signaled through cAMP $\left(\mathrm{G}_{\mathrm{\alpha s}}\right)$ and exhibited typical OAR pharmacology [4], responding to octopamine and other known octopamine receptor agonists (clonidine, naphazoline, and phentolamine) (Figure 3A) in the nM range and not to other biogenic amines (dopamine and serotonin) (Table 1). As is typical for OARs, both genes exhibited a dampened response to tyramine (Figure 3A). The best agonists were clonidine and naphazoline, inducing a response in the $\mathrm{pM}$ range (Table 1), consistent with both receptors being $\alpha_{2}$-adrenergic receptor homologues [4]. The receptor responses to octopamine were also inhibited by synthetic antagonists of OARs (chlorpromazine, cyproheptadine, metaclopramide, and promethazine) (Figure $3 \mathrm{~B}$ and Table 1), further indicating that both AgOAR45A and AgOAR45B are functional OARs [4].

To examine the ability of AgOAR45A and AgOAR45B to signal through $\mathrm{G}_{\alpha \mathrm{i}}$, cAMP activity was assessed in the presence of forskolin in response to octopamine and naphazoline (agonists of OARs) and inhibitory activity was not detected for either receptor. To monitor $\mathrm{Ca}^{2+}$ signals $\left(\mathrm{G}_{\alpha \mathrm{q}}\right.$ signaling), HEK293 reporter cells stably expressing either $A g O A R 45 A$ or $A g O A R 45 B$ were preloaded with the calcium sensitive dye Fluo-4 before stimulation with octopamine. Both receptors also signal through $G_{\alpha q}$ (see Additional file 5).

\section{Discovery of five potential binding modes of AgOAR45B}

As no functional differences between AgOAR45A and A5OAR45B were detected, in silico characterization and screening was performed with only AgOAR45B. Homology models of AgOAR45B were generated for both the inactive (antagonist-bound) and active (agonist-bound) conformations. As expected, the largest differences between the inactive and active conformations were the intracellular and extracellular loops as well as both $\mathrm{C}$ and $\mathrm{N}$-termini, including the beginning of the TM1 region. In general, differences in the TM regions of the two conformations were relatively small (see Additional file 6). The models were refined by MD simulations with the most active agonist (octopamine) and antagonist (promethazine) of the test set for the active and inactive conformations, respectively. For each of the four starting positions, MD was run using two different seeds, resulting in eight simulations total. For the inactive conformation, promethazine bound in two different conformations to D100 (Figure 4A and B). Similarly, three different binding modes were found for octopamine after 20 ns MD simulation (Figure 4C-E). Three very different ligand positions were obtained from the simulation of the active 


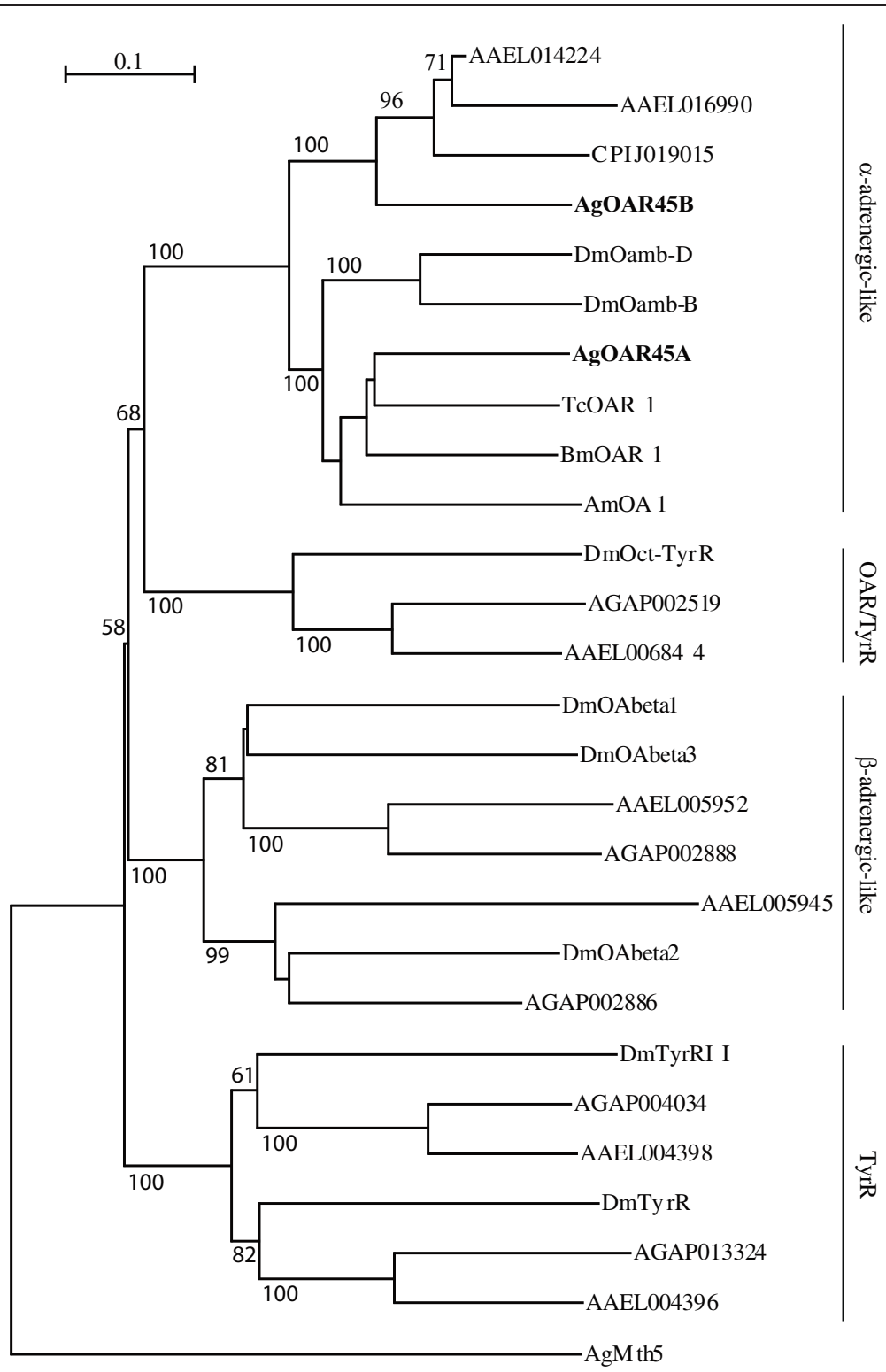

Figure 1 Phylogenetic relationship of Anopheles gambiae AgOAR45A and AgOAR45B with other octopamine (OAR) and tyramine (TyrR) insect receptors. Sequences from An. gambiae (AGAP000045, AGAP002519, AGAP002888, AGAP002886, AGAP004034, AGAP013324; Ae. aegypti (AAEL004398, AAEL004396, AAEL006844, AAEL014224, AAEL016990, AAEL005952, AAEL005945), and Cu. quinquefasciatus (CPIJ019015) were downloaded from VectorBase. Sequences from D. melanogaster [DmTyrRII (CG16766), DmTyrR (CG7431), DmOct-TyrR (CG7485), DmOamb-B (CG3856-RB), DmOamb-D (CG3856-RD), DmOAbeta1 (CG6919), DmOAbeta3 (CG42244) and DmOAbeta2 (CG33976)] were downloaded from FlyBase. Additional sequences, from Bombyx mori [BmOA1 (NP_001091748.1)], Apis mellifera [AmOA1 (NP_001011565.1)], and Tribolium [TcOAR1 (DAA64496.1)] were downloaded from GenBank. The alignment was performed on complete amino acid sequences and calculated using the ClustalX 2.1 [49]. The tree was constructed using ClustalX 2.1, and the neighbor-joining algorithm with a bootstrap value of 1,000. Numbers on branches are the percentage of bootstrap support for each branch node, only those above $50 \%$ are represented on the trees. The scale represents the rate of amino acid substitution per site. AgOAR45A and Ag OAR45B are in bold.

conformation, with octopamine binding to either both D100 and S206 (Figure 4C), S210 only (Figure 4D), or simultaneously binding to S206, S210 and E161 (Figure 4E).

Each of the antagonist and agonist-bound homology models were simulated for $20 \mathrm{~ns}$ in a combination membrane and water environment that approximately reproduces the protein's biological surroundings. The simulations were stopped every $10 \mathrm{~ns}$ and the stability of the ligands studied. After 20 ns, two of the antagonist-bound active site conformations and three of the agonist-bound active site conformations stabilized. The other simulations were continued for another $10 \mathrm{~ns}$, but no new stable conformations were observed. The stabilization of the active sites of the five generated AgOAR45B conformations was further confirmed 


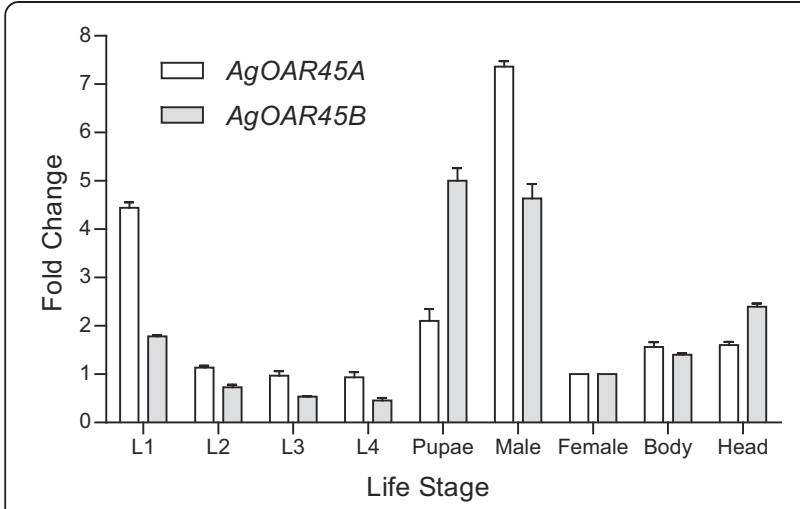

Figure 2 Expression levels of AgOAR45A and AgOAR45B.

Quantitative PCR analysis of An. gambiae AgOAR45A (grey) and AgOAR45B (white) at different life stages, including larvae (L1-L4), pupae, whole body adult females and males, adult female heads and thorax/abdomen. Data are expressed as fold change relative to whole females. Means \pm SD of three independent experiments are presented. via calculation of the RMSDs of the MD simulation trajectories compared to their initial conformations (see Additional file 7). The ligand and active site for each conformation were found to have a very stable interaction for at least ten full nanoseconds (10-20 ns maximum RMSD fluctuation $<1 \AA$ ).

\section{Mutagenesis of AgOAR45B}

To identify the receptor amino acid residues important for activity, we performed site-directed mutagenesis analysis of the residues indicated by the homology modelling to be important for binding. A number of studies have identified important residues vital for GPCR agonist binding activity and have indicated the importance of an aspartic acid in TM3 (D100) and serine residues in TM5 (S206 and S210) [10-12] and this has been shown to be true for $\alpha$-adrenergic-like octopamine receptors [46]. MD simulations of AgOAR45B suggested that a glutamic acid (E161) residue in TM4 may also play a role in ligand recognition. Therefore, AgOAR45B mutants were constructed changing each of these residues to alanine (A) residues by site-directed mutagenesis. The role of the
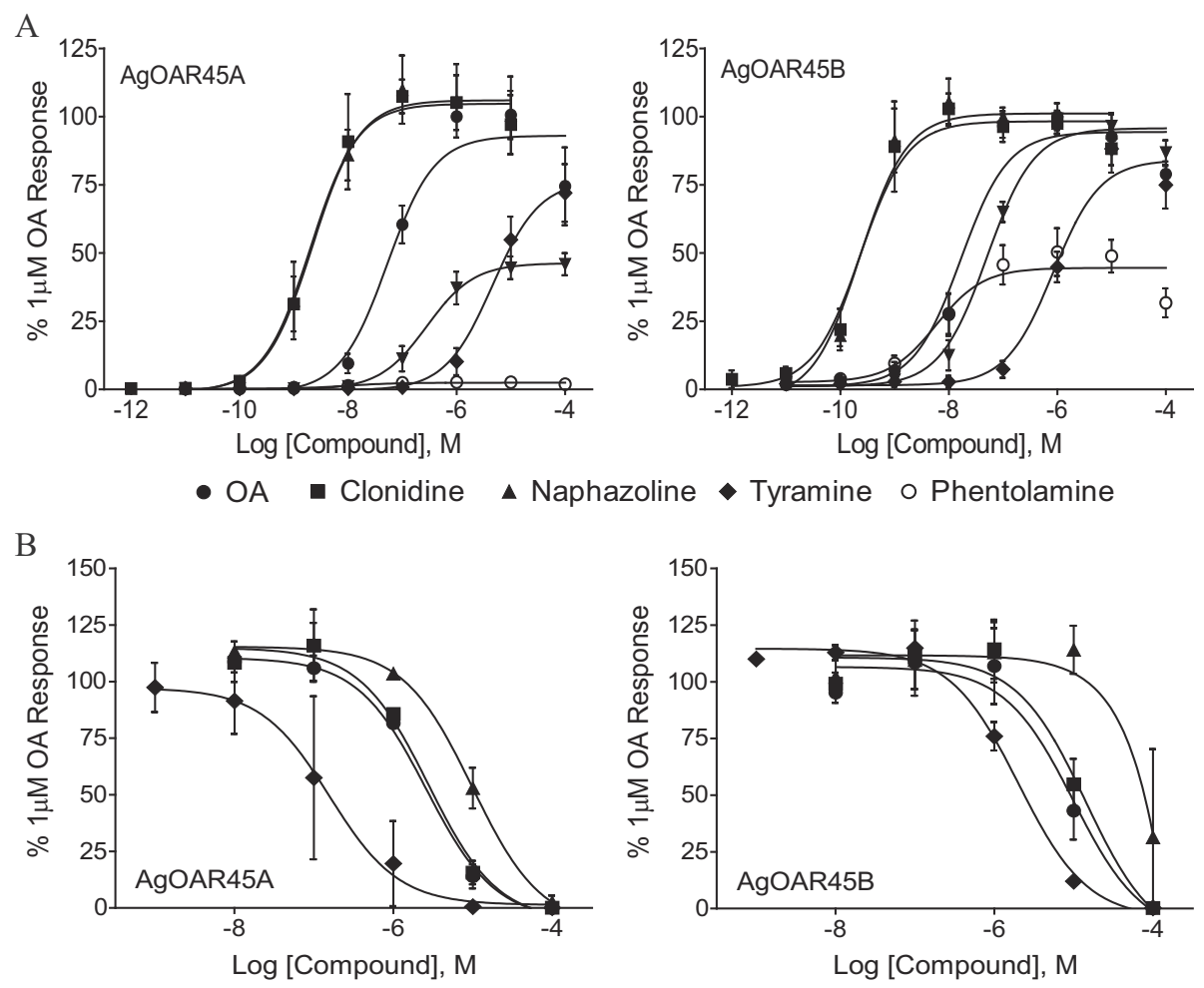

- Chlorpromazine - Cyproheptadine $\Delta$ Metoclopramide Promethazine

Figure 3 Pharmacological characterization of Anopheles gambaie octopamine receptors AgOAR45A and AgOAR45B in HEK293-CRE-Luc cells. A. Dose response curves of AgOAR45A and AgOAR45B to known octopamine receptor agonists [octopamine (OA; closed circles), clonidine (closed boxes), naphazoline (closed triangles), tyramine (closed diamonds), phentolamine (open circles)]. B. Dose response curves of known octopamine receptor antagonists [chlorpromazine (closed circles), cyproheptadine (closed boxes), metaclopramide (closed triangles), promethazine (closed diamonds) of AgOAR45A and in the presence of $1 \mu \mathrm{M}$ octopamine. Data are expressed as \% $1 \mu \mathrm{M}$ octopamine response. Means \pm SD of three independent experiments are presented. 


\begin{tabular}{|c|c|c|}
\hline Receptor & AgOAR45A & AgOAR45B \\
\hline Agonist & \multicolumn{2}{|c|}{$\mathrm{EC}_{50}(\mathrm{nM})$} \\
\hline Octopamine & 53 & 17 \\
\hline Clonidine & 2 & 0.24 \\
\hline Naphazoline & 2.2 & 0.23 \\
\hline Tolazoline & 280 & 5 \\
\hline Tyramine & 4600 & 840 \\
\hline Phentolamine & 10 & 5.8 \\
\hline Serotonin & $>50,000$ (inactive) & $>50,000$ (inactive) \\
\hline Dopamine & $>50,000$ (inactive) & $>50,000$ (inactive) \\
\hline Antagonist & \multicolumn{2}{|c|}{$\mathrm{IC}_{50}(\mu \mathrm{M})$} \\
\hline Chlorpromazine & 2.6 & 9.9 \\
\hline Cyproheptadine & 2.8 & 14 \\
\hline Metaclopramide & 9.8 & $>50$ (inactive) \\
\hline Promethazine & .16 & 2.0 \\
\hline
\end{tabular}

aspartic acid (D100) and the glutamic acid (E161) by mutating these residues into their non-charged amino acids (asparagine $(\mathrm{N})$ and glutamine $(\mathrm{Q})$, respectively) was further assessed. Mutated forms of $\operatorname{AgOAR} 45 \mathrm{~B}$ were generated via site-directed mutagenesis and transiently expressed into the GloResponse ${ }^{\mathrm{Tm}} \mathrm{CRE}-l u c 2 P$ HECK293 reporter cell line. Dose response curves for octopamine were generated for each single and double mutant receptor (Figure 5). Mutagenesis of either the aspartic acid or the glutamic acid resulted in loss of AgOAR45B to responsiveness to octopamine (Figure 5A). Single mutant analyses revealed that the aspartic acid residue is essential for octopamine activity as mutagenesis of this residue to either an alanine or an asparagine completely abolished octopamine reactivity. The glutamic acid residue, however, is marginally important for the response (Figure 5A). The results also indicate that while both serine residues participate in the octopamine response, S210 is more important than S206 (Figure 5B).

\section{Discovery of four antagonist-binding scaffolds}

Two different antagonist conformations of AgOAR45B were screened against the ZINC library. Of the resulting 1,500 compounds from each of the two conformations, the top 700 compounds from each were analysed and separated these compounds into four primary bindingscaffold groupings which were determined based on the hydrogen bonding of the D100 residue found in the active site of the protein with one of four binding sites in each antagonist: protonated piperazine, protonated imidazole, protonated $-\mathrm{NR}_{2}$, and a fourth group with other potential binding scaffolds (see Additional file 8).

\section{Discovery of four agonist-binding scaffolds}

Similarly, three different conformations were used to model the agonist conformation. Of the resulting 1,500 compounds from each of the three conformations, the top 250 compounds from each were analysed and separated these compounds into four binding-scaffold groupings. These scaffolds were determined based on the hydrogen bonding of the D100 residue found in the active site of the protein with one of four binding sites in each agonist: protonated piperazine, protonated $-\mathrm{NR}_{2} /-\mathrm{NR}_{3},-\mathrm{NH}-\mathrm{R}-$ $\mathrm{NH}$ - (where D100 interacts with both nitrogens), and a fourth group with other potential binding scaffolds (see Additional file 9). The other active portion of the compounds appeared to either hydrogen bond with one or the other of the serine residues in the TM5 region, or potentially did $\pi$ stacking with some of the benzene ringcontaining residues in the TM6 region. Both of these residues have been shown to move in the presence of agonists $[27,28]$ and were thus both considered. Scaffolds where the agonist hydrogen bonded only to the E161 residue were not considered due to E161's low importance in the activation of the protein as determined from the mutagenesis assays (Figure 5).

\section{New potential insecticides}

From the 2,150 compounds assessed from the two antagonist-bound and three agonist-bound conformations, a total of 70 compounds were chosen and purchased. These compounds were tested in vitro against AgOAR45B

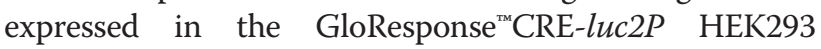
reporter cell line to determine their efficacy in being antagonists or agonists. Ultimately, 21 antagonists, 17 weak antagonists, two agonists, and five weak agonists were identified, while 25 of the 70 compounds were not active in the reporter assay (see Additional files 10 and 11).

Eight of the compounds identified from the antagonist conformation ZINC screen (46140767, 46140913, 1279 0591, 15671138, 12434335, 4148879, 24757679, and 4243 7778 ) inhibited the $1 \mu \mathrm{M}$ octopamine response of the $\mathrm{AgO}$ AR45B receptor reporter assay by greater than $40 \%$, however, these compounds decreased the viability of the HEK293 reporter cells (see Additional files 10 and 11, superscript c).

Additional chemistries that resulted in a reduction in receptor stimulation to $1 \mu \mathrm{M}$ octopamine less than two standard deviations ( $<80 \%$ activity) were chosen for further characterization. Four compounds (65552607, 32860047, 27417161, and 9274026) exhibited a dose response for at least two concentrations. Dose-response curves were generated for 9274026 and 9274026-like compounds (6791 891, 9273955, 15725975, and 9274053) using the AgOA R45B receptor reporter assay. As the high concentrations of the 9274026-like compounds were insoluble, $\mathrm{IC}_{50}$ values could not be accurately assessed, however, the results 


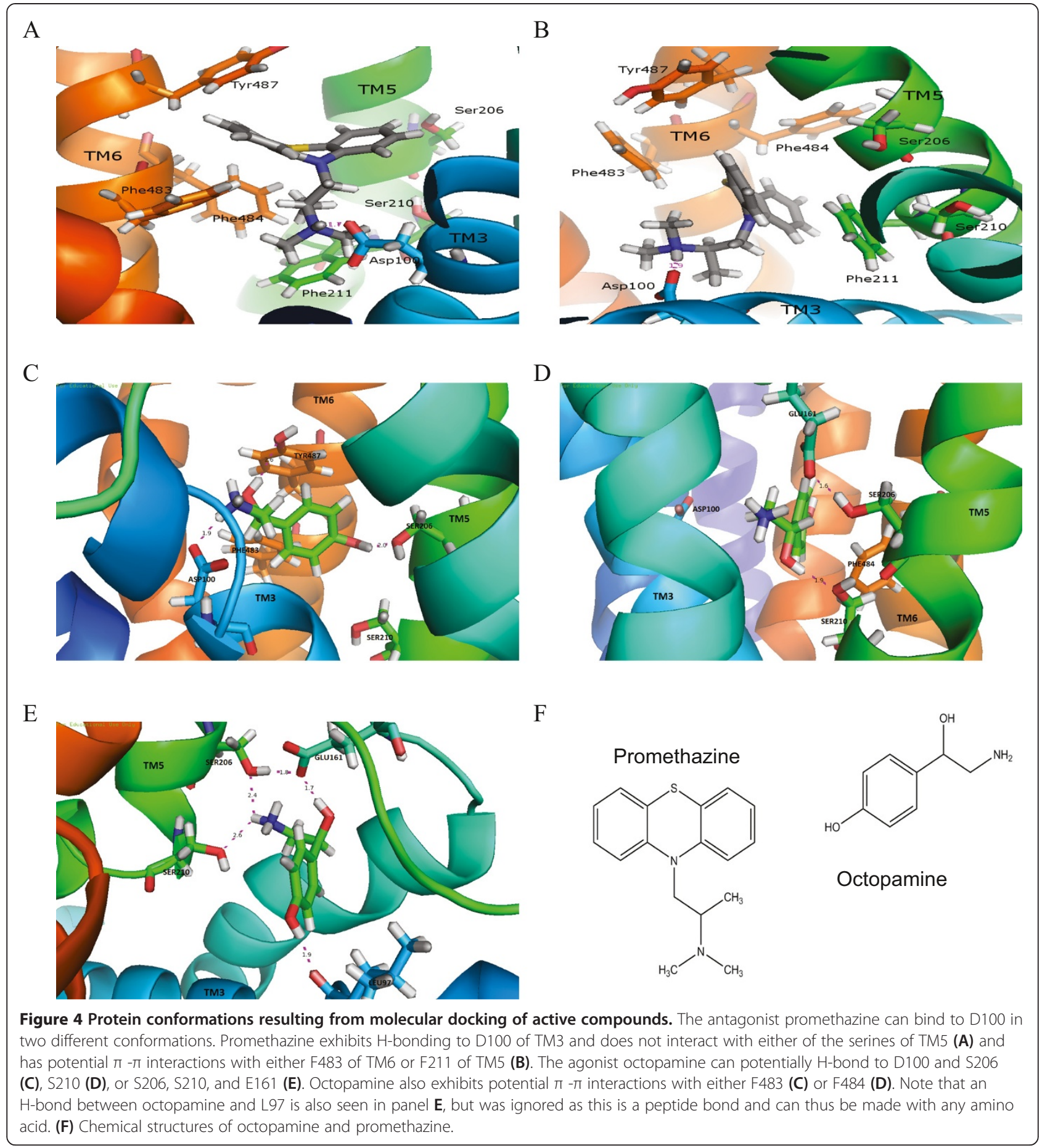

indicate that all four of the 9274026-like compounds were able to inhibit the response of AgOAR45B to $1 \mu \mathrm{M}$ octopamine as well as the 9274026 compound (11\% of the 1 $\mu \mathrm{M}$ octopamine response; $\mathrm{IC}_{50} 13.2 \mu \mathrm{M}$; see Additional files 10 and 11). Similarly, five compounds similar to 27417161 (12535485, 32843433, 12484738, 00200688, and 02721570) were selected and dose-response curves were generated. Two of the compounds, 00200688 and 1248
4738, induced responses similar to 27417161 ( 40\% inhibition; see Additional files 10 and 11).

Four compounds identified from the agonist-bound confirmation behaved as weak agonists: 00441586-13\%, $48194443-3 \%, 48345912-8.3 \%$, and $33313914-4.2 \%$ of octopamine response (see Additional files 10 and 11). Five compounds similar to 48345912 (48354000, 48353672, 4853678, 40118665, and 12984232) were selected for 


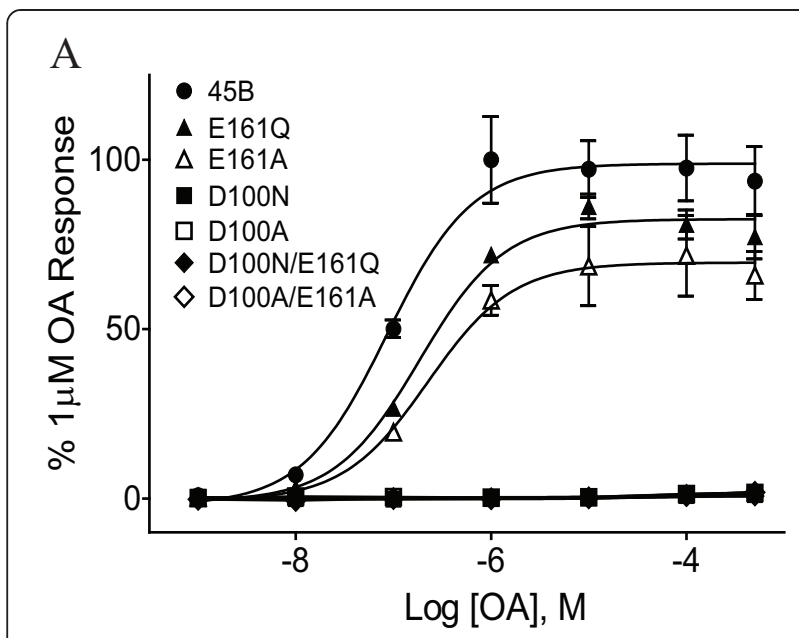

$\mathrm{B}$

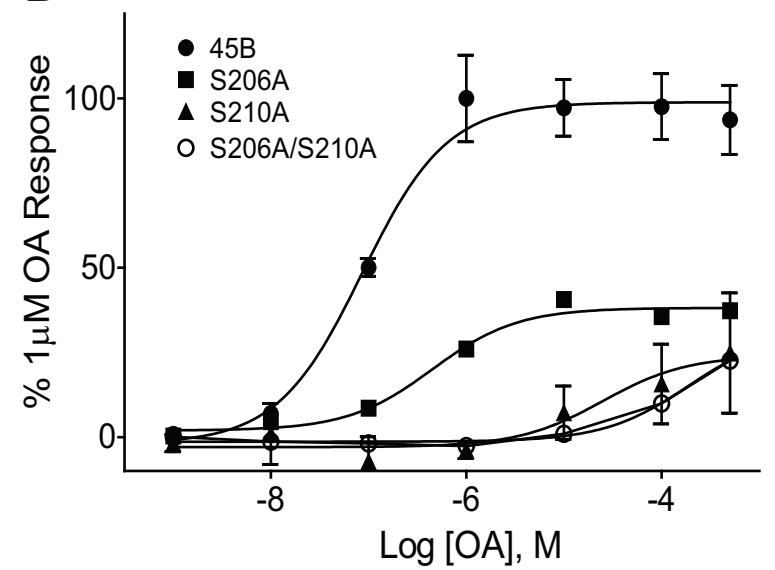

Figure 5 Pharmacological characterization of AgOAR45B mutants. A. Dose response curves of optopamine E161Q (closed triangles), E161A (open triangles), D100N (closed squares), D100A (open squares), D100N/E161Q (closed diamonds), and D100A/E161A (open diamonds) double mutants. B. Dose response curves of optopamine S206A (closed boxes), S210A (closed triangles) and S206A/S210A double mutants (open circles). The same control wild-type AgOAR45B (closed circles) data is presented in both A and B. Data are expressed as \% $1 \mu \mathrm{M}$ octopamine response. Means \pm SD of three independent experiments are presented.

further testing. One of the compounds, 48353678, had agonistic activity higher than to 48345912 (93.4\% of octopamine response). These compounds were further assessed for antagonistic activity in the presence of $1 \mu \mathrm{M}$ octopamine and four compounds, 4834512, 48354000, 48353672, and 40118665, were better antagonists than agonists. Compound 48353678, however, exhibited no antagonistic activity (see Additional files 10 and 11).

Two additional chemistries identified in the original in silico screens (10883478 and 02643656) were selected. One compound, 02643656, exhibited agonist activity slightly less than 48353678. Compound 10883478 behaved as an antagonist, inhibiting the response of AgOAR45B to
$1 \mu \mathrm{M}$ octopamine by $83 \%$. The 10883478 scaffold was further explored by selecting ten compounds (61715350, 58171610, 58007135, 61715380, 7205872, 11207230, $12547680,39985747,12794746$, and 32322962) from the ZINC library that were similar to 10883478 . Only one (12547680) had antagonistic activity similar to the parent compound. None of the compounds exhibited any agonistic activity (see Additional files 10 and 11).

To assess agonist and antagonist binding to AgOAR45B the best two agonists (48353678 and 02643656) and three antagonists (10883478, 40118665, and 48345912) were selected and competition binding assays with ${ }^{3} \mathrm{H}$-Yohimbine were performed. All the compounds exhibited affinity to AgOAR45B that was similar to octopamine (i.e. exhibited Ki values within an order of magnitude of the octopamine Ki) (Table 2).

To assess insecticidal activity, all 70 compounds in an Ae aegypti larvicide bioassay were tested.

Only 4 compounds induced any mortality. In the initial assay $(19922690,12434335,28695014)$ induced a small amount (14-21\%) of mortality compared to $0 \%$ in the DMSO alone group at 24 hours and so were not tested further. Compound 10883478, however, induced substantial mortality, killing $70.5 \pm 20.5 \%$ of the larvae at 24 hours.

\section{Discussion}

Octopamine is the most abundant biogenic amine in invertebrates and is virtually absent in vertebrates, making this pathway an attractive target for insecticide development with potential low toxicity in vertebrates. Often thought of as the functional equivalent of vertebrate norepinephrine, octopamine is an invertebrate neuromodulator, neurohormone and neurotransmitter. The octopaminergic system is involved in a variety of insect physiological processes, including locomotion, memory, mating, and egg laying [47]. Interestingly, cocaine, a natural insecticide, is believed to potentiate octopaminergic neurotransmission [48]. In addition, the formamidine pesticides, chlordimeform and amitraz are thought to act as octopamine agonists [49]. Here, two An. gambiae octopamine receptors, AgOAR45A and AgOAR45B were functionally characterized. Through in silico screening approaches one scaffold that holds

Table 2 Binding and larvicide activity of ZINC compounds

\begin{tabular}{lllll}
\hline ZINC COMPOUND & $\mathrm{Ki}(\boldsymbol{\mu M})$ & $\mathrm{EC}_{\mathbf{5 0}}(\boldsymbol{\mu M})$ & $\mathrm{IC}_{50}(\boldsymbol{\mu M})$ & $\begin{array}{l}\text { Larval bioassay } \\
(\% \text { mortality })\end{array}$ \\
\hline $\mathrm{OA}$ & 7.7 & 0.017 & - & $\mathrm{nd}^{*}$ \\
10883478 & 34.2 & - & 55.3 & 74 \\
02643656 & 14.9 & 5.6 & - & $\mathrm{nd}$ \\
40118665 & 56.7 & - & 17.6 & $\mathrm{nd}$ \\
48353678 & 2.7 & 22.0 & - & $\mathrm{nd}$ \\
48345912 & 6 & - & 4.0 & $\mathrm{nd}$ \\
\hline
\end{tabular}

*Not detectable. 
promise for the development of a novel insecticide was identified.

We identified six full-length OAR/TyrR genes in the An. gambiae genome. Phylogenetic analysis revealed two $\beta_{2}$-adrenergic like receptors, two TyrR, one OA/Tyr receptor and a gene that encodes two alternatively spliced OAR transcripts, $A g O A R 45 A$ and $A g O A R 45 B$. All seven predicted transcripts shared the characteristic sevem TM domains characteristic of GPCRs. AgOAR45A and $A g O A R 45 B$ are splice variants sharing the first three exons and differing only with the fourth exon. Both predicted proteins possess the conserved aspartic acid in TM3 and two of three conserved serines known to mediate binding of GPCRs to biogenic amines [50]. While identical for the first 246 amino acids, $A g O A R 45 A$ and $A g O A R 45 B$ only share $32 \%$ identity (46\% similarity) at the $\mathrm{C}$-terminal end of the protein. These amino acids encode the third intracellular loop and the C-terminus of the receptor thought to mediate the interaction between the GPCR and G-proteins [51,52], invoking the possibility that the two splice variants couple to different G-Protein signaling pathways. However, these results revealed that AgOAR45A and AgOAR45B, both signal through $\mathrm{Ca}^{++}$and cAMP, similar to their homologous proteins, DmOamb-B and DmOamb-D, in Drosophila [16,53].

GPCRs generally have three closely grouped serine residues found in TM5 that are located in the binding site. AgOAR45B, however, retains only two of these serine residues. The results demonstrated that D100, S206 and S210 are important in the agonistic function of AgOAR45B to octopamine, similar to what has been demonstrated for other GPCR-Ligand combinations [9-11]. It should be noted however, that while the serine residues do appear to be important, the function of the protein is not solely dependent on these residues. Even with both serine residues replaced, AgOAR45B can still respond to higher concentrations of octopamine (Figure 5B). This observation suggests that another mechanism may be involved in the activation of this protein. One hypothesis is that the ligand may also be involved in $\pi-\pi$ interactions (i.e. the attractive, non-covalent interactions that occur between aromatic rings with some of the aromatic residues) in the TM6 region (Figure 4). It seems possible that as the TM6 region moves towards the TM3 region in order to form these interactions, that the cytoplasmic end of the TM6 region moves outward. This hypothesis is supported by previous work in which the cytoplasmic end of TM6 region is shown to move outward when the protein is activated $[27,28]$.

One interesting scaffold group found to be effective contained mostly antagonists (48345912, 48354000 and 40118665) and one agonist (48353678). It is likely that this difference is due primarily to the length of the short chain to the pyridine group of 48353678 , as compared to the longer chain to the indoline group of 48353672 . It is possible that the shorter chain of 48353678 causes the active site to be more condensed and be more like the active conformation, while the longer chain of 48353672 allows the active site to assume a more open and inactive conformation. Docking of these compounds shows that both ligands can bind to the same residues in a similar conformation, yet the longer chain of 48353672 appears to be forcibly constrained and would preferably be extended, allowing the pocket to open.

Two of the common scaffolds that found docking to both agonist-bound and antagonist- bound AgOAR45B conformations, i.e., protonated piperazine and protonated $-\mathrm{NR}_{2}$, were also found in another study performed with the dopamine D3 receptor [54]. However, none of the active compounds identified contain protonated piperazine and only two contain protonated $-\mathrm{NR}_{2}$ (see Additional files 10 and 11). This, along with the presence of another scaffold that was not reported in the dopamine D3 receptor study, i.e., protonated imidazole (which 10883478 contains), indicates that there may be specificity for AgOAR45B to certain compound types.

Four of the compounds exhibited activity against mosquito larvae in an initial assay. Interestingly, these compounds were among the best antagonists and exhibited between 14 and $70.5 \%$ in a mosquito larval bioassay. Three (10883478, 12434335 and 28695014) of the four were imidazole derivatives with different carbon and nitrogen substitution patterns. Interestingly, a compound from the same scaffold as 10883478 that also exhibited potent antagonist activity was not active in the larvicide bioassay. Possibly, the ortho methyl substitution of 10883478 makes this compound less susceptible to metabolism.

\section{Conclusions}

Here, two An. gambiae OARs were functionally characterized and a computational approach coupled with experimental methods was utilized to discover possible lead compounds for the development of novel insecticides. We identified two AgOAR45B agonists and twenty-one antagonists, with one antagonist exhibiting substantial larvicide activity in a mosquito bioassay. However, we were unable to directly attribute OAR antagonism to the killing activity because of potential off target effects at the high concentrations utilized. Further studies on all the compounds to enhance potency, increase stability, and assess possible off target effects are needed. In addition, refinement of the computational models utilized for virtually screening will increase the possibility of identifying additional compounds for development. Running longer conventional MD simulations for ensuring optimal states is computationally impractical; however, accelerated molecular dynamics (aMD) will allow simulations on the millisecond timescale in only hundreds of nanoseconds [48]. In addition, a 
solved crystal structure of AgOAR45B would facilitate a more accurate model of the protein for the in silico discovery of lead compounds.

\section{Additional files}

\section{Additional file 1: Primer sequences for mutagenesis of $A g O A R 45 B$. \\ Additional file 2: Diagram of the basic computational-experimental} workflow.

Additional file 3: Alignment of AgOAR45B (AGAP000045B) with human b2-adrenergic receptor (NP_000015). Black boxes indicate active site residues. AgOAR45B exhibits a $67 \%$ sequence active site identity ( $73 \%$ similarity) to the $\beta_{2}$-adrenergic receptor active site.

Additional file 4: Genomic organization of AgOAR45A and AgOAR45B. AGAP000045 region downloaded from VectorBase. Maroon boxes indicate protein-coding regions. B) Protein alignment of the two proteins performed using Geneious software. Red boxes indicate transmembrane regions. Note low identity after position 250. C) Hydropathy Plot for AGAP000045B.

Additional file 5: AgOAR45A and AgOAR45B Gaq signaling. Dose response curves of AgOAR45A (circles) and AgOAR45B (squares) to known different concentrations of octopamine. Data are expressed as the change in fluorescence intensity $\left(F_{\max }-F_{\min }\right)$ divided by the background fluorescence intensity $\left(F_{\min }\right) \% 1 \mu \mathrm{M}$ octopamine response. Means \pm SD of three independent experiments are presented.

\section{Additional file 6: AgOAR45B homology models obtained from} I-TASSER. Blue indicates the inactive conformation while orange indicates the active conformation. Promethazine, shown in green, indicates the active site. Both sides of the proteins are presented, with (A) showing TM 1-5 and (B) showing TM 5-7, 2, and 1. Total Protein RMSD: $10 \AA$. TM-region RMSD: $2 \AA$.

Additional file 7: RMSDs of MD simulation trajectories compared to their respective initial conformations. (A) RMSD MD simulation yielding antagonist-bound conformation presented in Figure $4 \mathrm{~A}$, max flux $=0.82 \AA$. (B) RMSD MD simulation yielding antagonist-bound conformation presented in Figure 4B, max flux $=0.86 \AA$. (C) RMSD MD simulation yielding agonist-bound conformation presented in Figure 4C, max flux $=0.38 \AA$. (D) RMSD MD simulation yielding agonist-bound conformation presented in Figure 4D, max flux $=0.47 \AA \AA$. (E) RMSD MD simulation yielding agonist-bound conformation presented in Figure $4 \mathrm{E}$, max flux $=0.72 \AA$. Max flux values reported are the largest RMSD differences in the 10-20 ns simulation trajectories for the respective runs.

Additional file 8: Scaffolds obtained from antagonist-bound screens. The most commonly observed interactions with D100 in the virtual screen were (A) protonated piperazine, (B) protonated imidazole and, (C) protonated -NR2. Figure (D) shows one of the other potential interactions that were observed.

Additional file 9: Scaffolds obtained from agonist-bound screens. The most commonly seen interactions with D100 in the virtual screen were (A) protonated piperazine, (B) protonated $-\mathrm{NR}_{2} /-\mathrm{NR}_{3}$ and, (C) -NH-R$\mathrm{NH}$ - (where D100 interacts with both nitrogens). Figure (D) shows one of the other potential interactions that were observed.

Additional file 10: Activity of Zinc compounds tested in vitro. Additional file 11: Zinc chemistries purchased and tested in the AgOAR45B reporter assay.

\section{Abbreviations}

OAR: Octopamine receptor; GPCR: G-protein coupled receptor; MD: Molecular dynamics; TM: Transmembrane; GPCR: Quantitative PCR; RMSD: Root-mean-square deviation; POPC: 1- palmitoyl-2oleoylphosphatidylcholine; ns: Nanoseconds; ps: Picoseconds; fs: Femtoseconds.

\section{Competing interests}

The authors declare that they have no competing interests.

\section{Authors' contributions}

KWK performed the computational model preparation and simulation work, analysed and interpreted the data (primarily computational and some experimental). GLE supervised docking experiments and aided in interpretation of data. DAS performed the biological science experiments, along with JW and MFF and analysed the resulting data. LDM analysed the compound structures to determine improved compounds to purchase and test. JAI and MAM conceptualized the study, supervised the progress of the project, and aided in interpretation of the data. KWK, GLE, DAS, and MAM participated in writing the manuscript. All authors have read and approved the final version of the manuscript.

\section{Acknowledgements}

We are grateful to Gwen Stayback and Mariha Wadsworth for their insights and efforts in maintaining the mosquito colonies. We are thankful for Dr. Roger Sunahara and his laboratory at the University of Michigan for initial discussion on this project and their help in the development of the radioligand binding assay procedure. This work was supported by the Department of Defense Telemedicine and Advanced Technology Research Center (TATRC) awards \#W81XWH-10-1-0085 and W81XWH-11-1-0415 and the University of Notre Dame Chemistry-Biochemistry-Biology Interface (CBBI) Program, supported by NIH Training Grant T32GM075762. The funders had no role in the study design, data collection and analysis, decision to publish, or preparation of the manuscript.

\section{Author details}

'Department of Computer Science and Engineering, University of Notre Dame, Notre Dame, IN, USA. ${ }^{2}$ Department of Chemistry and Biochemistry, University of Notre Dame, Notre Dame, IN, USA. ${ }^{3}$ Department of Biological Sciences, Eck Institute for Global Health, University of Notre Dame, Notre Dame, IN, USA.

Received: 6 August 2014 Accepted: 3 November 2014 Published: 18 November 2014

\section{References}

1. Vaccines CM, Health BI, Prevention BHPD: Vaccines Against Malaria: Hope in a Gathering Storm. 1996.

2. Ashley EA, Dhorda M, Fairhurst RM, Amaratunga C, Lim P, Suon S, Sreng S, Anderson JM, Mao S, Sam B, Sopha C, Chuor CM, Nguon C, Sovannaroth S, Pukrittayakamee S, Jittamala P, Chotivanich K, Chutasmit K, Suchatsoonthorn C, Runcharoen R, Hien TT, Thuy-Nhien NT, Thanh NV, Phu NH, Htut Y, Han KT, Aye KH, Mokuolu OA, Olaosebikan RR, Folaranmi OO, et al: Spread of artemisinin resistance in Plasmodium falciparum malaria. N Engl J Med 2014, 371:411-423.

3. WHO: World Malaria Report 2010. Geneva: World Health Organization; 2010.

4. Ohta H, Ozoe Y: Molecular signallin, pharmacology, and physiology of octopamine and tyramine receptors as potential insect pest control targets. Adv Insect Physiol 2014, 46:73-166.

5. Roeder T: Octopamine in invertebrates. Progr Neurobiol 1999, 59:533-561.

6. Casida JE, Durkin KA: Neuroactive insecticides: targets, selectivity, resistance, and secondary effects. Annu Rev Entomol 2013, 58:99-117.

7. Filmore D: It's a GPCR world. Modern Drug Dis 2004, 7:24-28.

8. Overington JP, Al-Lazikani B, Hopkins AL: How many drug targets are there? Nat Rev Drug Discov 2006, 5:993-996.

9. Tautermann CS, Pautsch A: The implication of the first agonist bound activated GPCR X-ray structure on GPCR in silico modeling. ACS Med Chem Lett 2011, 2:414-418.

10. Congreve M, Langmead CJ, Mason JS, Marshall FH: Progress in structure based drug design for $\mathrm{G}$ protein-coupled receptors. J Med Chem 2011, 54:4283-4311.

11. Strader CD, Candelore MR, Hill WS, Sigal IS, Dixon RA: Identification of two serine residues involved in agonist activation of the beta-adrenergic receptor. J Biol Chem 1989, 264:13572-13578.

12. Wiens BL, Nelson CS, Neve KA: Contribution of serine residues to constitutive and agonist-induced signaling via the D2S dopamine receptor: evidence for multiple, agonist-specific active conformations. Mol Pharmacol 1998, 54:435-444.

13. Grohmann L, Blenau W, Erber J, Ebert PR, Strünker T, Baumann A: Molecular and functional characterization of an octopamine receptor from honeybee (Apis mellifera) brain. J Neurochem 2003, 86:725-735. 
14. Ohtani A, Arai Y, Ozoe F, Ohta H, Narusuye K, Huang J, Enomoto K, Kataoka $\mathrm{H}$, Hirota A, Ozoe Y: Molecular cloning and heterologous expression of an alpha-adrenergic-like octopamine receptor from the silkworm Bombyx mori. Insect Mol Biol 2006, 15:763-772.

15. Bischof $L$, Enan EE: Cloning, expression and functional analysis of an octopamine receptor from Periplaneta americana. Insect Biochem $\mathrm{Mol}$ Biol 2004, 34:511-521.

16. Balfanz S, Strunker T, Frings S, Baumann A: A family of octopamine [corrected] receptors that specifically induce cyclic AMP production or Ca2+ release in Drosophila melanogaster. J Neurochem 2005, 93:440-451.

17. Maqueira B, Chatwin H, Evans PD: Identification and characterization of a novel family of Drosophila beta-adrenergic-like octopamine G-protein coupled receptors. J Neurochem 2005, 94:547-560.

18. Behura SK, Gomez-Machorro C, Debruyn B, Lovin DD, Harker BW, Romero-Severson J, Mori A, Severson DW: Influence of mosquito genotype on transcriptional response to dengue virus infection. Funct Integr Genomics 2014,

19. White BJ, Kundert PN, Turissini DA, Van Ekeris L, Linser PJ, Besansky NJ: Dose and developmental responses of Anopheles merus larvae to salinity. $J$ Exp Biol 2013, 216:3433-3441.

20. Megy K, Emrich SJ, Lawson D, Campbell D, Dialynas E, Hughes DST, Koscielny G, Louis C, Maccallum RM, Redmond SN, Sheehan A, Topalis P, Wilson D, VectorBase Consortium: VectorBase: improvements to a bioinformatics resource for invertebrate vector genomics. Nucleic Acids Res 2012, 40:D729-734.

21. Yarnitzky T, Levit A, Niv MY: Homology modeling of G-protein-coupled receptors with X-ray structures on the rise. Curr Opin Drug Discov Develop 2010, 13:317-325.

22. Roy A, Kucukural A, Zhang Y: I-TASSER: a unified platform for automated protein structure and function prediction. Nat Protoc 2010, 5:725-738.

23. Roy $A, X u$ D, Poisson J, Zhang $Y$ : A protocol for computer-based protein structure and function prediction. J Vis Exp 2011, 57:3259-3268.

24. Zhang Y: I-TASSER server for protein 3D structure prediction. BMC Bioinformatics 2008, 9:40.

25. Humphrey W, Dalke A, Schulten K: VMD: visual molecular dynamics. J Mol Graph 1996, 14:33-38. 27-38.

26. I-Tasser Suite. http://zhanglab.ccmb.med.umich.edu/I-TASSER/.

27. Rasmussen SG, Choi HJ, Fung JJ, Pardon E, Casarosa P, Chae PS, Devree BT, Rosenbaum DM, Thian FS, Kobilka TS, Schnapp A, Konetzki I, Sunahara RK, Gellman SH, Pautsch A, Stevaert J, Weis WI, Kobilka BK: Structure of a nanobody-stabilized active state of the $\beta(2)$ adrenoceptor. Nature 2011, 469:175-180.

28. Rasmussen SGF, DeVree BT, Zou Y, Kruse AC, Chung KY, Kobilka TS, Thian FS, Chae PS, Pardon E, Calinski D, Mathiesen JM, Shah ST, Lyons JA, Caffrey M, Gellman SH, Steyaert J, Skiniotis G, Weis WI, Sunahara RK, Kobilka BK: Crystal structure of the $\beta 2$ adrenergic receptor-Gs protein complex. Nature 2011, 477:549-555.

29. Brooks BR, Brooks CL, Mackerell AD, Nilsson L, Petrella RJ, Roux B, Won $Y$, Archontis G, Bartels C, Boresch S, Caflisch A, Caves L, Cui Q, Dinner AR, Feig M, Fischer S, Gao J, Hodoscek M, Im W, Kuczera K, Lazaridis T, Ma J, Ovchinnikov V, Paci E, Pastor RW, Post CB, Pu JZ, Schaefer M, Tidor B, Venable RM, et al: CHARMM: the biomolecular simulation program. J Comput Chem 2009, 30:1545-1614.

30. Case DA, Darden TA, Cheatham TE, Simmerling CL, Wang J, Duke RE, Luo R, Crowley MRCW, Walker RC, Zhang W, Merz KM, Wang B, Hayik S, Roitbert A, Seabra G, Kolossvary I, Wong KF, Paesani F, Vanicek J, Wu X, Brozell SR, Steinbrecher T, Gohlke H, Yang L, Tan C, Mongan J, Homak V, Cui G, Mathews DH, Seetin MG, et al: Amber 11. 2010.

31. Duan Y, Wu C, Chowdhury S, Lee MC, Xiong G, Zhang W, Yang R, Cieplak P, Luo R, Lee T, Caldwell J, Wang J, Kollman P: A point-charge force field for molecular mechanics simulations of proteins based on condensed-phase quantum mechanical calculations. J Comput Chem 2003, 24:1999-2012.

32. Wang J, Wolf RM, Caldwell JW, Kollman PA, Case DA: Development and testing of a general amber force field. J Comput Chem 2004, 25:1157-1174.

33. Wang J, Wang W, Kollman PA, Case DA: Automatic atom type and bond type perception in molecular mechanical calculations. J Mol Graph Model 2006, 25:247-260.

34. Phillips JC, Braun R, Wang W, Gumbart J, Tajkhorshid E, Villa E, Chipot C, Skeel RD, Kalé L, Schulten K: Scalable molecular dynamics with NAMD. J Comput Chem 2005, 26:1781-1802.

35. Kraken. http://www.nics.tennessee.edu/computing-resources/kraken.
36. Suite 2011: Schrödinger Suite 2011 Protein Preparation Wizard; Epik version 2.2, Schrödinger, LLC, New York, NY, 2011; Impact version 5.7, Schrödinger, LLC, New York, NY, 2011; Prime version 3.0, Schrödinger, LLC, New York, NY, 2011. New York, NY.

37. Madhavi Sastry G, Adzhigirey M, Day T, Annabhimoju R, Sherman W: Protein and ligand preparation: parameters, protocols, and influence on virtual screening enrichments. J Comput Aided Mol Des 2013, 27:221-234.

38. Suite 2011: Maestro, version 9.2, Schrödinger, LLC, New York, NY, 2011. New York, NY.

39. The PyMOL Molecular Graphics System, Version 1.3r1, Schrödinger, LLC. 2010.

40. Suite 2011: Glide, version 5.7, Schrödinger, LLC, New York, NY. New York, NY; 2011.

41. Friesner RA, Banks JL, Murphy RB, Halgren TA, Klicic JJ, Mainz DT, Repasky MP, Knoll EH, Shelley M, Perry JK, Shaw DE, Francis P, Shenkin PS: Glide: a new approach for rapid, accurate docking and scoring. 1. Method and assessment of docking accuracy. J Med Chem 2004, 47:1739-1749.

42. Friesner RA, Murphy RB, Repasky MP, Frye LL, Greenwood JR, Halgren TA, Sanschagrin PC, Mainz DT: Extra precision glide: docking and scoring incorporating a model of hydrophobic enclosure for protein-ligand complexes. J Med Chem 2006, 49:6177-6196.

43. Halgren TA, Murphy RB, Friesner RA, Beard HS, Frye LL, Pollard WT, Banks L: Glide: a new approach for rapid, accurate docking and scoring. 2. Enrichment factors in database screening. J Med Chem 2004, 47:1750-1759.

44. Suite 2011: LigPrep, version 2.5, Schrödinger, LLC, New York, NY. New York, NY; 2011.

45. Irwin JJ, Sterling T, Mysinger MM, Bolstad ES, Coleman RG: ZINC: a free tool to discover chemistry for biology. J Chem Inf Mod 2012, 52:1757-1768.

46. Huang J, Hamasaki T, Ozoe F, Ohta H, Enomoto K, Kataoka H, Sawa Y, Hirota A, Ozoe $Y$ : Identification of critical structural determinants responsible for octopamine binding to the alpha-adrenergic-like Bombyx mori octopamine receptor. Biochemistry 2007, 46:5896-5903.

47. Verlinden H, Vleugels R, Marchal E, Badisco L, Pfluger HJ, Blenau W, Broeck $\mathrm{JV}$ : The role of octopamine in locusts and other arthropods. J Insect Physiol 2010, 56:854-867.

48. Nathanson JA, Hunnicutt EJ, Kantham L, Scavone C: Cocaine as a naturally occurring insecticide. Proc Natl Acad Sci U S A 1993, 90:9645-9648.

49. Gole JW, Orr GL, Downer RG: Interaction of formamidines with octopamine-sensitive adenylate cyclase receptor in the nerve cord of Periplaneta americana L. Life Sci 1983, 32:2939-2947.

50. Strader CD, Fong TM, Graziano MP, Tota MR: The family of G-protein-coupled receptors. FASEB J 1995, 9:745-754.

51. Bourne HR: How receptors talk to trimeric G proteins. Curr Opin Cell Biol 1997, 9:134-142.

52. Wess J: G-protein-coupled receptors: molecular mechanisms involved in receptor activation and selectivity of G-protein recognition. FASEB J 1997, 11:346-354.

53. Han KA, Millar NS, Davis RL: A novel octopamine receptor with preferential expression in Drosophila mushroom bodies. J Neurosci 1998, 18:3650-3658

54. Carlsson J, Coleman RG, Setola V, Irwin JJ, Fan H, Schlessinger A, Sali A, Roth $B L$, Shoichet BK: Ligand discovery from a dopamine D3 receptor homology model and crystal structure. Nat Chem Biol 2011, 7:769-778.

doi:10.1186/1475-2875-13-434

Cite this article as: Kastner et al:: Characterization of the Anopheles gambiae octopamine receptor and discovery of potential agonists and antagonists using a combined computational-experimental approach. Malaria Journal 2014 13:434. 